\title{
Correction: Unique CDR3 epitope targeting by CAR-T cells is a viable approach for treating T-cell malignancies
}

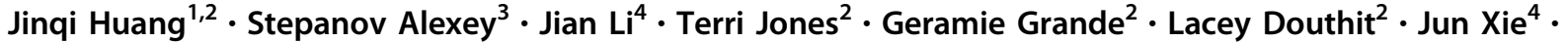 \\ Danna Chen ${ }^{5}$ Xiaolei $\mathrm{Wu}^{6} \cdot$ Maschan Michael ${ }^{7} \cdot$ Changchun Xiao ${ }^{4,8} \cdot$ Jiangning Zhao ${ }^{9} \cdot$ Xuehua Xie $^{10} \cdot$ Jia Xie $^{2}$. \\ Xiao Lei Chen $\mathbb{1}^{4} \cdot$ Guo Fu ${ }^{4} \cdot$ Gabibov Alexander ${ }^{3}$. Chi-Meng Tzeng ${ }^{1,11}$
}

Published online: 16 May 2019

(c) Springer Nature Limited 2019

\section{Correction to: Leukemia}

https://doi.org/10.1038/s41375-019-0455-3

published online 8 April 2019

In the original version of this article the author name Xiaolei Chen was published incorrectly. This has been corrected to Xiao Lei Chen.
A single corresponding author was shown in the original article. This has been corrected to include the following four authors as joint corresponding authors: Xiao Lei Chen (Cx12015@xmu.edu.cn); Guo Fu (guofu@xmu.edu.cn); Gabibov Alexander (gabibov@mx.ibch.ru); Chi-Meng Tzeng (cmtzeng@xmu.edu.cn).

This has now been corrected in both the PDF and HTML versions of the Article.
These authors contributed equally: Jinqi Huang, Stepanov Alexey, Jian Li.

The original article can be found online at https://doi.org/10.1038/ s41375-019-0455-3.

Xiao Lei Chen

Cx12015@xmu.edu.cn

$\triangle \mathrm{Guo} \mathrm{Fu}$ guofu@xmu.edu.cn

$\triangle$ Gabibov Alexander gabibov@mx.ibch.ru

$\triangle$ Chi-Meng Tzeng cmtzeng@xmu.edu.cn

1 Translational Medicine Research Center (TMRC), School of Pharmaceutical Sciences, Xiamen University, Xiamen, China

2 Department of Chemistry, The Scripps Research Institute, La Jolla, CA 92037, USA

3 M.M. Shemyakin and Yu.A. Ovchinnikov Institute of Bioorganic Chemistry, Russian Academy of Sciences, Miklukho-Maklaya Str., 16 /10, Moscow 117997, Russia
4 State Key Laboratory of Cellular Stress Biology, Innovation Center for Cell Signaling Network, School of Life Sciences, Xiamen University, Xiamen, Fujian, China

5 The Affiliated Hospital of Putian University, Putian, Fujian, China

6 ProteinT Biotechnology Ltd. Co. Tianjin Airport Free Trade Zone, Tianjin, China

7 Dmitrii Rogachev Federal Research Center for Pediatric Hematology, Oncology and Immunology, Samory Mashela Str. 1, Moscow 117997, Russia

8 Department of Immunology and Microbiology, The Scripps Research Institute, La Jolla, CA 92037, USA

9 Department of Hematology, Zhongshan Hospital of Xiamen University, Xiamen 361004 Fujian, China

10 Department of Oncology, The first hospital of Putian City, Putian 351100 Fujian, China

11 School of Pharmacology, Nanjing Tech University, Nanjing, China 